\title{
Knee Effusion after Arthroscopic Partial Meniscectomy: Prospective Study Comparing Preventing Methods
}

\author{
Andrés Pakuts, Luis Martin \\ School of Medicine, Pontifical Catholic University of Chile, Santiago, Chile \\ Email:1fmartin@uc.cl
}

How to cite this paper: Pakuts, A. and Martin, L. (2019) Knee Effusion after Arthroscopic Partial Meniscectomy: Prospective Study Comparing Preventing Methods. Open Journal of Orthopedics, 9, 152-158. https://doi.org/10.4236/ojo.2019.98016

Received: July 8, 2019

Accepted: August 6, 2019

Published: August 9, 2019

Copyright $\odot 2019$ by author(s) and Scientific Research Publishing Inc. This work is licensed under the Creative Commons Attribution International License (CC BY 4.0).

http://creativecommons.org/licenses/by/4.0/

(c) (i) Open Access

\begin{abstract}
Background: Knee joint effusion is a common adverse event after arthroscopic procedures and its prevention is crucial to achieve better clinical outcomes, such as pain relief, improved range of movement and global satisfaction. Objective: The aim of this study is to compare joint effusion after partial arthroscopic meniscectomy between a common postoperative management and a short period in deep flexion. Methods: A prospective study randomizes 62 patients who underwent arthroscopic partial meniscectomy into 2 groups of postoperative care: compression elastic bandage with elevation for 24 hours (compression group) versus immediate postoperative deep flexion for 2 hours (flexion group). Clinical evaluation of effusion was made at 24 hours, 7 days and 1-month post-surgery. Results: Overall at 24 hours patients showed 53\% absent effusion, 35\% minimum, $11 \%$ had mild or more severe effusion. Effusion rates in the compression group were $13 \%, 10 \%$ and $0 \%$; in the flexion group $10 \%, 6.4 \%$ and $0 \%$, at 24 hours, 7 days and 1 month, respectively. There was no statistically significant difference between them $(\mathrm{p}>$ 0.05). Conclusion: In the studied population, position of the knee in deep flexion had similar influence in effusion outcomes than a standard widely used treatment, offering an alternative in the postoperative care of the patients.
\end{abstract}

\section{Keywords}

Arthroscopy, Effusion, Prevention, Postoperative Knee Position

\section{Introduction}

One of the postoperative clinical signs that allow us to evidence an intraarticular 
process secondary to intraoperative trauma or intra articular injuries is effusion. It is known that articular effusion causes postoperative inconvenience such as pain; also influences recovery process, tissue repair, range of movement (ROM), muscle activation, clinical evolution and global satisfaction of the procedure [1] [2] [3] [4]. Knee arthroscopy is a minimally invasive procedure in which the whole joint sustains a traumatic process of variable intensity due to penetration of surgical instruments and fluid. It is associated to reduce hospital stay, less morbidity, earlier recovery and less radiologic changes in comparison to open surgery [5]. Depending on the type of procedure performed, there will be different degrees of inflammation and trauma, being bleeding the most common cause of effusion [6] [7]. Partial meniscectomy consists in removing injured meniscal tissue aiming to preserve as much healthy tissue as possible in an attempt to reduce pain and long-term chondral damage [8]. This procedure is indicated in different types of meniscal lesions resulting in the most common of all orthopedic surgeries [9].

Reducing knee effusion after arthroscopic procedures is an important operative goal in order to achieve earlier and a comfortable postoperative period. A frequent, postoperative care to avoid effusion after partial meniscectomy, is installing compressive elastic bandage, leaving the knee in elevation and semi flexion during the postoperative period until hospital discharge. This management takes time in the operating room, leaves the patient with the bandage and in a reduced mobility position that is often uncomfortable especially in the overnight patients.

Ewing and associates [10] described that the mean intra articular pressure during arthroscopy while the knee is in forced flexion is $159 \mathrm{mmHg}$ and in flexion just $78 \mathrm{mmHg}$ [1]. Based on these findings we postulate that an immediate postoperative period in deep flexion could prevent bleeding by raising the intraarticular pressure and thus diminishing the appearance of joint effusion. Accordingly, we compared the postoperative care previously mentioned with a postoperative period in flexion in order to determine the efficacy of this innovative position.

After thorough research of 2 medical databases (Medline \& Embase) by 2 independent reviewers we did not find any study regarding the effects of knee position on articular effusion post arthroscopic procedures.

\section{Methods}

We designed a randomized prospective study which included only patients who underwent partial meniscectomy by the same surgical team (one surgeon) in a private practice clinic between April $1^{\text {st }}, 2013$ and May 30 ${ }^{\text {th }}$, 2013. Partial meniscectomy was performed in a standard fashion using mechanical debridement with shaver and tissue stabilization with low grade radiofrequency. Patients who required additional procedures due to associated intraarticular pathology, requiring removal of bone, cartilage procedures or wide synovectomy, were ex- 
cluded. A total of 62 patients were recruited and randomized into two groups: "compression" group and "flexion" group. Compression group was managed with 1) compressive elastic bandage over a soft bandage installed previously from distal to proximal, to prevent excessive compression; and 2) elevated knee on a metallic platform that holds the leg leaving the hip and knee flexed in 45 degrees for approximately 24 hours. On the other hand, the flexion group was managed with deep knee flexion (about 110 degrees) in the immediate postoperative period for about 2 hours, usually matching with the time under spinal anesthetic effects. To maintain the knee in flexed position, the plantar surface of the ipsilateral foot was placed on the medial side of the contralateral knee with a bandage holding them together. A pillow was placed under the operated knee in order to prevent excessive hip abduction. After 2 hours the foot and knee were released allowing full motion and free positioning of the limb as tolerated (Figure 1).

Patients were evaluated clinically at 24 hours, 7 days and 1 month since surgery. Level of knee effusion was registered into 5 categories, as following: absent (0), minimal (1), mild (2), moderate (3) and severe (4). Sample size (at least 30 in each group) was decided in order to achieve normal distribution concordantly with the statistic tests that would be used for posterior analysis. Obtained data was analyzed using frequency, central tendency measures and contingency tables applying chi-squared adjusted by Mantel \& Haenszel with significance less than $5 \%(\mathrm{p}<0.05)$. This study has the respective ethical requirements and authorization of the director of medical center in charge of the patients included.

\section{Results}

After randomization, both groups had similar gender proportion: 15 men and 16 women in the compression group, and 14 men and 17 women in the flexion group. Mean age of both groups was 48 years. Age distribution was similar in

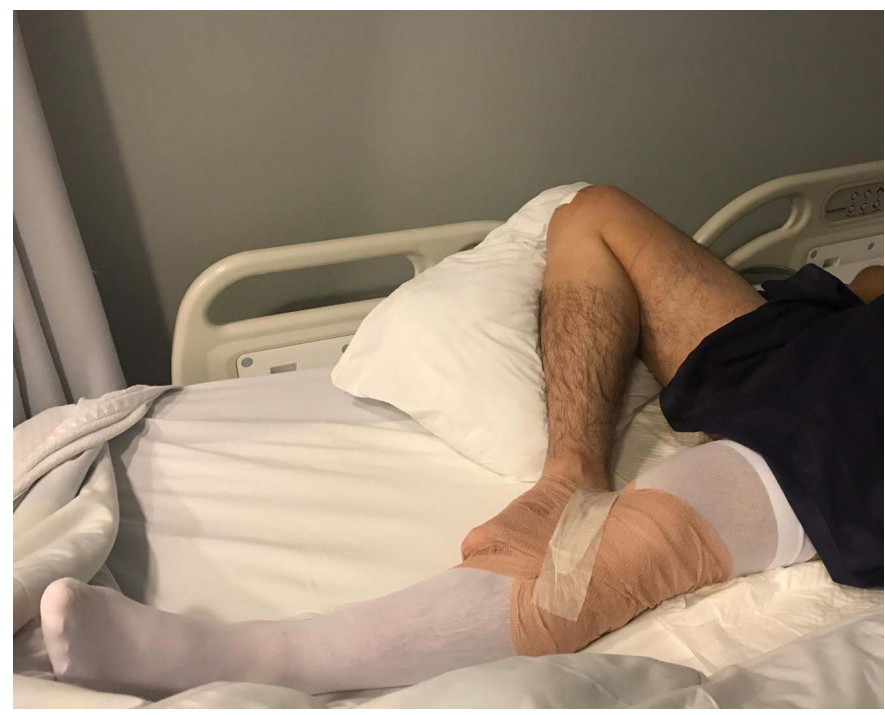

Figure 1. Flexion group patient position and management. 
both groups (Table 1). We did not find statistically significant differences in the level of effusion in neither the 24 hours, 7 days nor 1-month clinical evaluations. None of the patients had clinically significant effusion ( $\geq$ Mild) 1 month after surgery.

Effusion levels dropped importantly within a week and after a month clinically significant effusion was absent in both groups. Minimal effusion had the highest prevalence within all groups of effusion at 24 hours, reaching $35 \%$, more details regarding the level of effusion at 24 hours can be seen in Table 2. The prevalence of preoperative effusion was $3 \%$ in both groups. Effusion prevalence at 24 hours, 7 days and 1 month, in the flexion group was $10 \%, 6.4 \%$ and $0 \%$; and in compression group was $11 \%, 8 \%$ and $0 \%$, respectively. More details about prevalence of effusion can be seen in Table 3.

Patients of the flexion group had good tolerance of the deep knee flexion positioning (Figure 1) and none of the patients complained of discomfort. The above was addressed by the nurse in charge of the recovery and hospitalization, who was specifically instructed to release the position after 120 minutes after surgery. Also, neither of the patients in this group presented vascular abnormalities nor cutaneous lesions.

Table 1. Distribution according to age \& randomized group.

\begin{tabular}{ccc}
\hline Age Group (years) & Flexion Group & Compression Group \\
\hline $10-20$ & 1 & 0 \\
$20-30$ & 2 & 2 \\
$30-40$ & 6 & 5 \\
$40-50$ & 5 & 6 \\
$50-60$ & 6 & 8 \\
$60-70$ & 9 & 7 \\
$70-80$ & 1 & 0 \\
\hline
\end{tabular}

Table 2. Postoperative level of knee effusion at 24 hours.

\begin{tabular}{cc}
\hline Level of Effusion & Total \\
\hline Absent & $33(53 \%$, CI $40 \%-66 \%)$ \\
Minimum & $22(35 \%$, CI $24 \%-49 \%)$ \\
$\geq$ Mild & $7(11 \%$, CI $4.6 \%-22 \%)$ \\
Total & 62 \\
\hline
\end{tabular}

CI: confidence interval 95\%.

Table 3. Evolution of knee effusion ( $\geq$ Mild) according to randomized group.

\begin{tabular}{cccc}
\hline Time & Flexion group & Compression group & Total \\
\hline Preoperative & $1(3 \%$, CI $0.1 \%-17 \%)$ & $1(3 \%$, CI $0.1 \%-17 \%)$ & $2(3.2 \%$, CI $0.3 \%-11 \%)$ \\
24 hours & $3(10 \%$, CI $2 \%-26 \%)$ & $4(13 \%$, CI $3.6-30 \%)$ & $7(11 \%$, CI $4.6 \%-22 \%)$ \\
7 days & $2(6.4 \%$, CI $0.8 \%-21 \%)$ & $3(10 \%$, CI $2 \%-26 \%)$ & $5(8 \%$, CI $0.3 \%-18 \%)$ \\
1 month & 0 & 0 & 0 \\
\hline
\end{tabular}

CI: confidence interval $95 \%$. 


\section{Discussion}

Knee arthroscopy is a low morbidity procedure, in accordance with findings made by Lubowitz [11] who describes $82 \%$ unease walking rate at 7 days post-surgery in his 72 patient's cohort and Stetson and Tamplin [12] who found that prior to 20 days all their patients return to normal activity levels. Siemieniuk et al. [13] described a recovery period between 2 and 6 weeks post arthroscopy in which pain, effusion and limited function was present.

Alkan found rates around 70\% for mild or superior effusion the first week after arthroscopy with a confidence interval between $56 \%$ and $85 \%$ in the control group of his effusion prevention study [14]. Unlike our study, that shows just 8\% effusion prevalence one week after partial meniscectomy (confidence interval: $0.3 \%-18 \%$ ). This difference could be explained due to inclusion of patients with wide synovectomy, different surgical technique and postoperative care.

Tatari [15] in his study of 57 patients who underwent partial meniscectomy, found that none of them presented significant effusion at 4, 7 nor 15 days after the procedure. Concordantly, our group found similar results with $92 \%$ of patients not presenting significant effusion at 7 days post intervention. In addition, Chana's study has similar results, showing minimal or absent effusion at 2 weeks post arthroscopy [16].

Regarding therapeutic alternatives to prevent effusion, other authors compare the usual management versus postoperative drainage and other alternatives. They found no statistically significant difference between the usual care [14] [15] [17] [18]. Yakin and Rogers [19] in their study of comparison between conventional therapy and laser therapy, they obtained an effusion absent rate of $74.4 \%$ after one month in the conventional therapy group $(n=43$, confidence interval $59 \%-86 \%)$. Laser therapy had statistically significant lower rates of effusion but was not a cost-effective therapy. If we compare our results with Yakin and Rogers's trial, our rate of absent effusion at one month is $100 \%$. One of the pitfalls in our study is the lack of a control group that would need to be without any postoperative care procedures, elevation, compression nor special positioning. The latter was analyzed but not included due to ethical reasons.

Our study also has a limitation of presenting wide confidence intervals, similar to what was found in the literature regarding this issue. Methods found in the literature are varied, thus making difficult the comparison between them. Definitely this study is an innovative postoperative care as we didn't find studies that compared knee positioning in the immediate postoperative care to prevent effusion.

\section{Conclusion}

In conclusion, in the studied population, position of the knee in deep flexion for a short period in the immediate postoperative had similar results in effusion rates compared to a widely used compression with elastic bandage and elevation from the operating room until hospital discharge. Allowing full motion and free 
positioning of the limb, as tolerated, during the first 24 hours after surgery leaves the patient in a more comfortable state. Its clinical benefit is that we have an option in the early postoperative period that allows more freedom in body and motion without compromising effusion prevention rates. This should be particularly useful in patients with limited capability of staying prolonged periods in a dorsal decubitus position due to associated pathologies, like lumbar spine or hip problems.

\section{Acknowledgements}

Andrés Pakuts is specialist in orthopedic surgery by the Pontificial Catholic University of Chile \& Luis Martin is currently undergraduate in the same institution.

\section{Conflicts of Interest}

We declare no conflict of interests.

\section{References}

[1] Fabricant, P.D., Rosenberger, P.H., Jokl, P. and Ickovics, J.R. (2008) Predictors of Short-Term Recovery Differ From Those of Long-term Outcome after Arthroscopic Partial Meniscectomy. Arthroscopy. The Journal of Arthroscopic \& Related Surgery, 24, 769-778. https://doi.org/10.1016/j.arthro.2008.02.015

[2] Uzun, E., Misir, A., Kizkapan, T.B., Ozcamdalli, M., Akkurt, S. and Guney, A. (2017) Factors Affecting the Outcomes of Arthroscopically Repaired Traumatic Vertical Longitudinal Medial Meniscal Tears. Orthopaedic Journal of Sports Medicine, 5. https://doi.org/10.1177/2325967117712448

[3] Stalman, A.1., Tsai, J.A., Wredmark, T., Dungner, E., Arner, P. and Felländer-Tsai, L. (2008) Local Inflammatory and Metabolic Response in the Knee Synovium after Arthroscopy or Arthroscopic Anterior Cruciate Ligament Reconstruction. Arthroscopy: The Journal of Arthroscopic and Related Surgery, 24, 579-584. https://doi.org/10.1016/j.arthro.2007.12.010

[4] Niinikoski, J. and Einola, S. (1977) Postoperative Synovial Fluid Metabolic Response to Meniscectomy or Synovectomy. Acta Orthopaedica Scandinavica, 48, 129-137. https://doi.org/10.3109/17453677708985123

[5] Benedetto, K.P. and Rangger, C. (1993) Arthroscopic Partial Meniscectomy: 5-Year Follow-Up. Knee Surgery, Sports Traumatology, Arthroscopy, 1, 235-238. https://doi.org/10.1007/BF01560216

[6] Clifton, R., Haleem, S., McKee, A. and Parker, M.J. (2007) Closed Suction Surgical Wound Drainage after Anterior Cruciate Ligament Reconstruction: A Systematic Review of Randomised Controlled Trials. Knee, 14, 348-351. https://doi.org/10.1016/j.knee.2007.07.003

[7] Coupens, S.D. and Yates, C.K. (1991) The Effect of Tourniquet Use and Hemovac Drainage on Postoperative Hemarthrosis. Arthroscopy, 7, 278-282. https://doi.org/10.1016/0749-8063(91)90127-J

[8] Azar, F., Beaty, J. and Canale, S. (2017) Campbell's Operative Orthopaedics, Chapter 45. Elsevier, Philadelphia, PA, 2140-2156.

[9] Azar, F., Beaty, J. and Canale, S. (2017) Campbell's Operative Orthopaedics, Chap- 
ter 51. Elsevier, Philadelphia, PA, 2486-2546.

[10] Ewing, J., Noe, D., Kitaoka, H. and Askew, M. (1986) Intra-Articular Pressures during Arthroscopic Knee Surgery. Arthroscopy: The Journal of Arthroscopic \& Related Surgery, 2, 264-269. https://doi.org/10.1016/S0749-8063(86)80083-4

[11] Lubowitz, J.H., Ayala, M. and Appleby, D. (2007) Return to Activity after Knee Arthroscopy. Arthroscopy. The Journal of Arthroscopic \& Related Surgery, 24, 58-61.e4. https://doi.org/10.1016/j.arthro.2007.07.026

[12] Stetson, W.B. and Templin, K. (2002) Two- versus Three-Portal Technique for Routine Knee Arthroscopy. American Journal of Sports Medicine, 30, 108-111. https://doi.org/10.1177/03635465020300010301

[13] Siemieniuk, R.A., Harris, I.A., Agoritsas, T., Poolman, R.W., Brignardello-Petersen, R., Velde, S.V., et al. (2018) Arthroscopic Surgery for Degenerative Knee Arthritis and Meniscal Tears: A Clinical Practice Guideline. British Journal of Sports Medicine, 52, 313-313. https://doi.org/10.1136/bjsports-2017-j1982rep

[14] Alkan, K., Unay, K., Berjem, K., Güven, M. and Poyanli, O. (2011) Suction Drainage Influence on Knee Effusion Following Partial Meniscectomy with Partial Fat Pad or Synovium Resection. Acta Orthopaedica et Traumatologica Turcica, 45, 221-224. https://doi.org/10.3944/AOTT.2011.2545

[15] Tatari, H., Dervisbey, M., Muratli, K. and Ergor, A. (2005) Report of Experience in 190 Patients with the Use of Closed Suction Drainage in Arthroscopic Knee Procedures. Knee Surgery, Sports Traumatology, Arthroscopy, 13, 458-462. https://doi.org/10.1007/s00167-004-0537-1

[16] Chana, G.S. and Tubbs, N. (1981) Early Results of Arthroscopic Surgery of the Knee. Injury, 13, 227-229. https://doi.org/10.1016/0020-1383(81)90244-8

[17] Browett, J.P., Gibbs, A.N., Copeland, S.A. and Deliss, L.J. (1978) The Use of Suction Drainage in the Operation of Meniscectomy. The Journal of Bone and Joint Surgery, 60, 516-519. https://doi.org/10.1302/0301-620X.60B4.711799

[18] Bryan, R.S., Dickson, J.H. and Taylor, W.F. (1969) Recovery of the Knee Following Meniscectomy. The Journal of Bone and Joint Surgery, 51, 973-978. https://doi.org/10.2106/00004623-196951050-00014

[19] Yakin, D.E. and Rogers, V.P. (1999) Conventional Instrument vs. Laser-Assisted Arthroscopic Meniscectomy. Lasers in Surgery and Medicine, 25, 435-437. https://doi.org/10.1002/(SICI)1096-9101(1999)25:5<435::AID-LSM10>3.0.CO;2-Z 